\section{Expression of Caveolin-1 in tongue squamous cell carcinoma by quantum dots}

\author{
J. Xue, ${ }^{1}$ H. Chen, ${ }^{1}$ L. Diao, ${ }^{1}$ X. Chen, ${ }^{2}$ \\ D. Xia' \\ 'Department of Pathology, School of \\ Basic Medical Science, Wuhan University, \\ Wuhan, China \\ 2Department of Oral Pathology, School \\ of Stomatology, Wuhan University, \\ Wuhan, China
}

\section{Abstract}

Quantum dots (QDs) are a new class of fluorescent probes to detect biomarker expression. The role of caveolin-1 (Cav-1) in tongue squamous cell carcinoma (TSCC) is still unknown. This study aimed to investigate the expression profile of Cav-1 in carcinogenesis and development of TSCC by QDs immunofluorescence histochemistry (QDs-IHC) and discuss the relationship between the Cav-1 expression and the clinicopathological outcomes. QDs-IHC was used to detect Cav-1 expression in tissue microarrays including normal tongue mucosa (NTM; $n=10$ ), hyperplastic tongue mucosa (HTM; $n=10)$, tongue pre-cancer lesions (TPL; $n=15$ ) and primary tongue squamous cell carcinoma (PTSCC; $\mathrm{n}=61$ ). Correlations between the Cav-1 expression and clinicopathologic variables were evaluated statistically. Cells positive for Cav-1 were clearly detected and bright images were obtained in a fine, granular pattern at the cell membrane and cytoplasm using QDs-IHC. The rate of Cav-1 immunoreactivity increased progressively from NTM (0\%), HTM (0\%), TPL (36\%) to PTSCC (74\%). When compared with each other, there was statistical significance among PTSCC, TPL and NTM as well as among PTSCC, TPL and HTM. Moreover, Cav-1 expression level in PTSCC was correlated positively with clinical stage and histologic grade. QDsIHC could accurately detect protein location in tongue mucosa. An increased expression of Cav-1 in the stepwise carcinogenesis from NTM, HTM, TPL to PTSCC suggested that Cav1 might be an oncogene in the development of tongue squamous cell carcinoma.

\section{Introduction}

Oral squamous cell carcinoma (OSCC) is the most common malignant neoplasm of the oral mucosa; it ranks among the top twelfth most frequently diagnosed cancers worldwide. In China, tongue squamous cell carcinoma (TSCC) predominates more than $95 \%$ of OSCC and its incidence is increasing year by year. However, the pathogenesis of TSCC is not yet completely disclosed. Dysplastic lesions of the oral cavity range from mild to moderate and severe dysplasia, and the presence and degree of dysplasia are often used to predict malignant transformation. Moreover, the prognosis for most patients with OSCC, of course including TSCC, is usually related to clinical stage and histologic grade. ${ }^{1}$ Therefore, the identification of effective biologic markers that correlate with the stepwise of carcinogenesis and development in TSCC is very important to understand this neoplasm.

Caveolin-1 (Cav-1), a 21-24 kDa protein is the major structural component of caveolae, vesicular invaginations of the plasma membrane. Previous studies have implicated Cav-1 in the development of several human cancers, but it appears to play quite different roles in the development of these diseases: down-regulation of Cav-1 expression was observed in breast, lung, colon and ovarian cancer; ${ }^{2,3}$ other studies have reported that Cav-1 expression was up-regulated in some human cancers, such as prostate, esophagus, thyroid and pancreas cancers, and that this up-regulation was associated with metastases and poor prognosis. ${ }^{47}$ In OSCC as well, the role of Cav-1 still remains controversial: two research groups ${ }^{8,9}$ found that an increased Cav-1 expression plays an important role in carcinogenesis and development of OSCC. In contrast, some studies ${ }^{10}$ disclosed that the inactivation of Cav-1 by a mutation or by reduced expression might play a role in the pathogenesis of oral cancer, which indicated the value to explore Cav-1's biphasic functions in OSCC. To our best knowledge, there is no report about the relationship between Cav-1 expression and TSCC. Whether Cav-1 acts as oncogene or tumor suppressor gene in the development of TSCC derserves new insights.

Semiconductor quantum dots (QDs) are new nanocrystal semiconductor fluorophores consisting of a cadmium selenide core and zinc sulfide or cadmium sulfide shell. As described in previous publications, ${ }^{11}$ QDs can be used to target malignant tumors with high specificity, when conjugated with biomolecular affinity ligands, such as antibodies, peptides or small molecules. Due to their novel optical and electronic properties, such as high brightness, stability and simplified multiple target labeling, QDs recently attracted attention for their potential application in advanced biosensors, bioanalytical assays and cell imaging. ${ }^{12}$

In this report, using a new method QDs-IHC, we sought to investigate the expression of Cav-1 in normal tongue mucosa (NTM), hyper-
Correspondence: Honglei Chen, Department of Pathology, School of Basic Medical Science, Wuhan University, Wuhan 430071, China.

Tel.: +86.027.68759735 - Fax: +86.027.68758766

E-mail: xjlzcl@sina.com and chenhongleiwh@126.com

Key words: tongue squamous cell carcinoma, caveolin-1, quantum dots.

Contributions: JX and $\mathrm{HC}$ participated in the study design, QDs-IHC experiment, manuscript preparation and drafting. $\mathrm{LD}$ and XC helped samples collection and pathological diagnosis. JX carried out statistical analysis and DX performed QDs-IHC analysis and HE staining. All authors read and approved the final manuscript.

Received for publication: 18 January 2010.

Accepted for publication: 1 March 2010.

This work is licensed under a Creative Commons Attribution 3.0 License (by-nc 3.0).

(C) Copyright J. Xue et al., 2010

Licensee PAGEPress, Italy

European Journal of Histochemistry 2010; 54:e20 doi:10.4081/ejh.2010.e20

plastic tongue mucosa (HTM), tongue pre-cancer lesions (TPL) and TSCC, trying to elucidate the possible role of Cav-1 in carcinogenesis and development of TSCC.

\section{Materials and Methods}

\section{Histological examination and tissue microarray construction}

We evaluated 96 cores, including 61 cases of human TSCC, 10 cases of NTM, 10 cases of HTM and 15 cases of TPL using human tissue microarray (TMA) technology (Department of Oral Pathology, School of Stomatology, Wuhan University). The specimens and donors evaluated in this research were anonymous. Two board-certified pathologists re-confirmed the histopathologic features of these samples. Histological diagnosis and grades of differentiation were determined in accordance with the World Health Organization (WHO) criteria for oral tumors. ${ }^{13}$ Concerning the TSCC cases, all the samples were deriving from the tongue of 61 patients, 34 males and 27 females, with an average age of 54.8 years; the histological differentiation of TSCC was classified as "well", "moderate" or "poor". The tumor (T) stages were: T1-24, T2-34, T3-2, and T4-1. The neck (N) stages were: N0-48, N1-13. A total of 61 samples were stage M0 (metastasis 0 .)

All the tissues were taken from surgical resection prior to initial topical or systemic therapy. They were fixed in $10 \%$ neutral 
buffered formalin for 24 hours and processed using identical standard operating procedures (SOPs). Tissue samples were arranged in 6 columns of 16 rows for a total of 96 individual cores $(1.5 \mathrm{~mm}, 4 \mu \mathrm{m})$. Each slide has $>95 \%$ tissue core retention.

\section{Immunofluorence by quantum dots immunohistochemistry}

Four $\mu \mathrm{m}$-thickness TMAs were deparaffined in xylene and rehydrated in a graded series of ethanol. Immunofluorescence histochemistry was performed according to the manufacturer's instructions. Antigen retrieval was performed using citric acid (10 mM, pH 6.0) at $95^{\circ} \mathrm{C}$ for $10 \mathrm{~min}$ and left to cool for $60 \mathrm{~min}$. TMAs were incubated with $2 \%$ bovine serum albumin (BSA) (Sigma, St. Louis, M0, USA) buffer for $30 \mathrm{~min}$ at $37^{\circ} \mathrm{C}$. The slides were rinsed $3 \times$ with TBS-Tween 20 (TBS-T) (5 min). Sections were incubated with rabbit anti-human Cav-1 polyclonal antibody (1:300 dilution, Santa Cruz, CA, USA) for $2 \mathrm{~h}$ at $37^{\circ} \mathrm{C}$, followed by three washes with TBS-T ( $5 \mathrm{~min}$ ), then incubated with biotinylated goat anti-rabbit IgG (Zhongshan Golden Bridge Biotechnology C0, LTD., Beijing, China) for 30 min at $37^{\circ} \mathrm{C}$, and rinsed $3 \times$ with TBS-T (5 min). Slides were incubated with $2 \%$ BSA buffer for $10 \mathrm{~min}$ at $37^{\circ} \mathrm{C}$ before incubation with streptavidin-conjugated QDs605 (1:100, Wuhan Jiayuan Quantum Dot Co, LTD., Wuhan, Hubei, China) in $2 \%$ BSA for 30 min at $37^{\circ} \mathrm{C}$ and rinsed $3 \times$ with TBS-T ( $5 \mathrm{~min}$ ), the edges sealed with $90 \%$ glycerine (Sigma, St. Louis, M0, USA). The signal obtained from the labeling of cells was detected by using fluorescence microscopy. The QDs signals were shown to be target specific, red, bright, and photo stable. Cav-1 immunoreactivity was normally localized to fibroblasts and endothelial cells of blood vessels in all tissue specimens examined, which served as an internal quality control in QDs-IHC. TBS instead of Cav-1 primary antibody served as negative controls.

A reproducible semiquantitative analysis ${ }^{14}$ was used to evaluate the expression level of Cav-1 in tissue samples. The sections were initially scanned at low power, and areas with high and low staining intensity were selected. At each selected field, cells were classified with respect to staining intensity ranging from 0 to 4 , and the percentage of cells for each staining intensity was estimated by decimal equivalent ranging from 0 to 1 . A numerical value for Cav1 staining in each tissue section was then calculated by multiplying the fraction of cells at each staining intensity by the numerical value of that intensity, resulting in scores ranging from 0 to 4. Expression levels of each Cav-1 in tumor samples were graded based on the total score as follows: negative or low $=$ score $\leq 1$; moderate or high score $>1$. we defined the cutoff between score and score $>1$ because score 1 level was the most significant pathologic effect in clinicopathological features. Two observers evaluated independently the results of this method, without any knowledge of the clinical data. Positive cells for Cav-1 were cell membrane and cytoplasmic granular staining.

\section{Statistical analysis}

The $\chi^{2}$ test and Fisher's exact tests were used to compare differences between Cav-1 expression and different tongue lesion, which is a clinical pathological parameter of PTSCC. Statistical analysis was performed using SPSS version 13 software (SPSS Inc, Chicago, IL, USA). Type I error probability (2-sided) less than or equal to 0.05 was considered the level of significance for all statistical procedures.

\section{Results}

Immunofluorescence by QDs analysis revealed focal positive immunoreactivity for Cav-1 in normal squamous epithelium (Figure 1A) and hyperplastic squamous epithelium (Figure 1B). Among 15 dysplastic tissues, 5 showed moderate to high levels of Cav-1 expression (score $>1$ ), characterized by
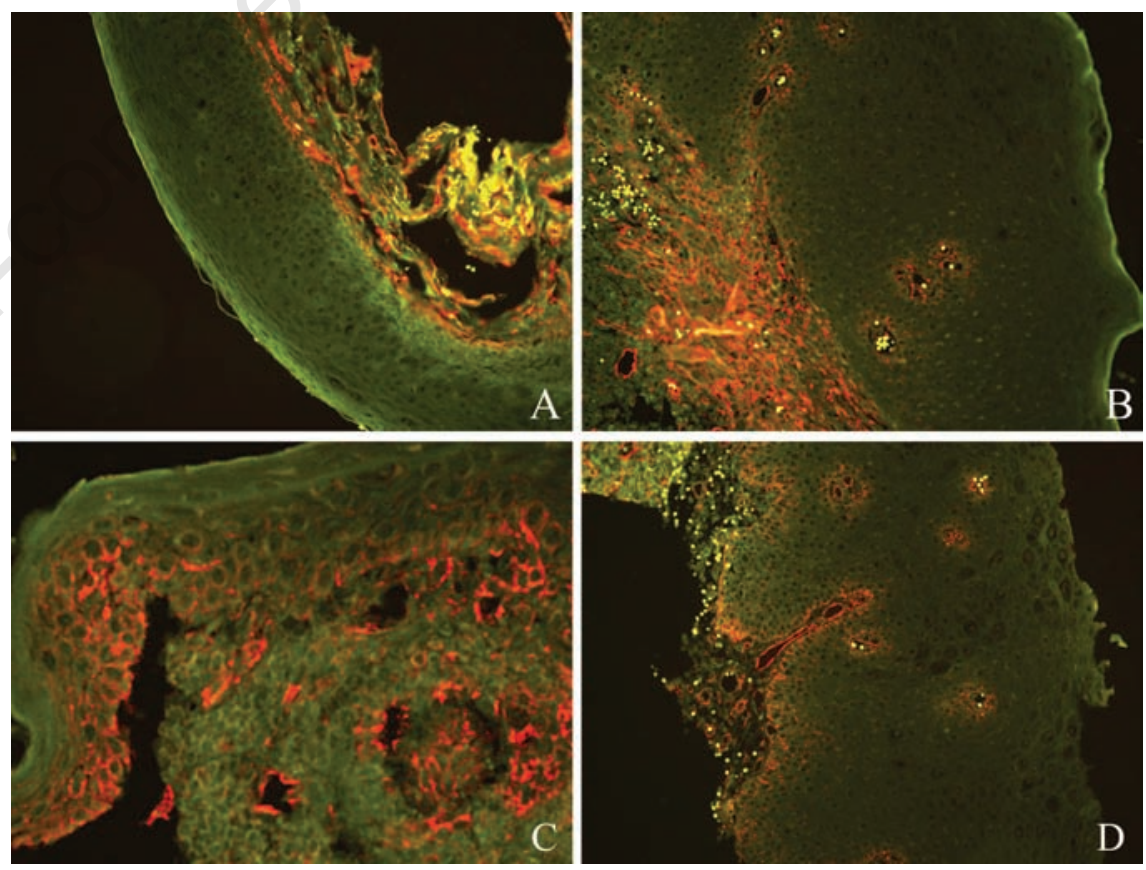

Figure 1. Cav-1 expression by QDs-IHC in normal, hyperplastic and displastic tongue mucosa. (A) Focal expression of Cav-1 in normal tongue mucosa (QDs-IHC $\times 200$ ). (B) Focal expression of Cav-1 in hyperplastic tongue mucosa and internal control showed a strong, diffuse, cytoplasmic staining pattern in the endothelial cells in the stroma vessels (QDs-IHC $\times 200)$. (C) Almost full thickness of epithelium in severely dysplasitic tongue mucosa is positive for Cav-1 (QDs-IHC $\times 400)$. (D) Cav-1 expression confined to basal or parabasal cells in mild or moderate dysplastic tongue mucosa $(\mathrm{QDs}-\mathrm{IHC} \times 200)$. 
Statistically, Cav-1 expression in the TPL and PTSCC showed significant increase compared with that of normal mucosa and hyperplastic mucosa respectively, $(\mathrm{P}<0.01$, Table 1). Cav-1 expression of PTSCC was also significantly higher compared with TPL $(\mathrm{P}<0.01$, Table 1). In PTSCC, the clinicopathologic variables are listed in Table 2. Statistical correlation was found between the enhanced Cav-1 expression and the degree of histological grades ("well", "moderate" and "poor", $\mathrm{P}<0.05)$. In addition, altered expression of Cav-1 was associated with the T stage and $\mathrm{N}$ classification. In contrast, there was no correlation between the positivity of Cav-1 expression and gender and age.

\section{Discussion}

Determination of the expression and spatial distribution of molecular epitopes, or antigens, in patient tissue specimens has substantially improved the pathologist's ability to classify disease processes. Certain tumor pathophysiologies are marked by characteristic increased or decreased expression of some proteins. The primary goals of tumor research are to identify these molecular factors, among which Cav-1 has attracted attention because of its altered expression patterns in tumour cells, which contribute to tumorigenesis and tumor progression. In this report, we have used a new QDs-IHC method to observe the expression of Cav-1 in stepwise carcinogenesis and development of TSCC on basic fluorescence microscope.

QDs, a novel inorganic fluorophores, may be cross-linked to biomolecules such antibodies, oligonucleotides or small molecule ligands to render them specific to biological targets. Due to their novel optical and electronic properties, the use of multicolor QDs probes is considered one of the most important and clinically relevant applications. They have been received as technological marvels with such characteristics able to greatly improve biological imaging and detection. A previous study ${ }^{15}$ revealed that QDs can be applied to formalin-fixed, paraffinembedded tissues; this technique achieves sensitivity and specificity levels that are sufficient for the potential application of known expression signatures to biopsy specimens in a semi-quantitative way, while the semi-automated nature of this method enables application to high-throughput studies. Some researchers ${ }^{16}$ demonstrated that QDs probe could be used for multiplexed profiling of molecular biomarkers, and ultimately for correlation with disease progression. Our previous study ${ }^{17}$ also indicated QDs-IHC is sensitive and specific in detecting the protein in paraf-
Table 1. Correlations of Cav-1 expression among normal tongue mucosa, hyperplastic tongue mucosa, tongue premalignant lesion and tongue squamous cell carcinoma.

\begin{tabular}{lccc} 
Groups & \multicolumn{2}{c}{ Cav-1 Score } & $\mathrm{P}$ \\
& $\leq 1.0$ & $>1$ & \\
Normal and hyperplastic tongue mucosa $^{\mathrm{a}}$ & 20 & 0 & $\mathrm{P}^{\mathrm{ab}}<0.01$ \\
Premalignant lesion $^{\mathrm{b}}$ & 10 & 5 & $\mathrm{P}^{\mathrm{bc}}<0.01$ \\
Tongue squamous cell carcinoma $^{\mathrm{c}}$ & 16 & 45 & $\mathrm{P}^{\mathrm{ac}}<0.01$ \\
\hline
\end{tabular}

$\mathrm{Pab}$, normal and hyperplastic tongue mucosa vs premalignant lesion; $\mathrm{Pbc}$, premalignant lesion vs tongue squamous cell carcinoma; $\mathrm{Pac}$, normal and hyperplastic tongue mucosa vs tongue squamous cell carcinoma.

Table 2. Relationship between clinicopathologic features and Caveolin-1 expression in primary tongue squamous cell carcinoma by QDs-IHC.

\begin{tabular}{|c|c|c|c|}
\hline \multirow[t]{2}{*}{ Variable } & \multicolumn{2}{|c|}{ Cav-1 Score } & \multirow[t]{2}{*}{$P$} \\
\hline & $\leq 1.0$ & $>1$ & \\
\hline \multicolumn{4}{|l|}{ Gender } \\
\hline Male & 7 & 27 & \multirow[t]{2}{*}{$P>0.05$} \\
\hline Female & 9 & 18 & \\
\hline \multicolumn{4}{|l|}{ Age } \\
\hline$\geq 60$ & 6 & 18 & \multirow[t]{2}{*}{$P>0.05$} \\
\hline$<60$ & 10 & 27 & \\
\hline \multicolumn{4}{|l|}{ T status* } \\
\hline T1 & 14 & 10 & \multirow[t]{2}{*}{$\mathrm{P}<0.05$} \\
\hline T2+T3+T4 & 2 & 35 & \\
\hline \multicolumn{4}{|l|}{$\mathrm{N}$ status $^{\circ}$} \\
\hline NO & 16 & 32 & \multirow[t]{2}{*}{$P<0.05$} \\
\hline N1 & 0 & 13 & \\
\hline \multicolumn{4}{|l|}{ Differentiation } \\
\hline Well & 15 & 18 & \multirow[t]{3}{*}{$P<0.05$} \\
\hline Moderate & 1 & 16 & \\
\hline Poor & 0 & 11 & \\
\hline
\end{tabular}

*Tumor stage, size or direct extent of the primary tumor; ${ }^{\circ}$ Neck stage, degree of spread to neck lymph nodes.
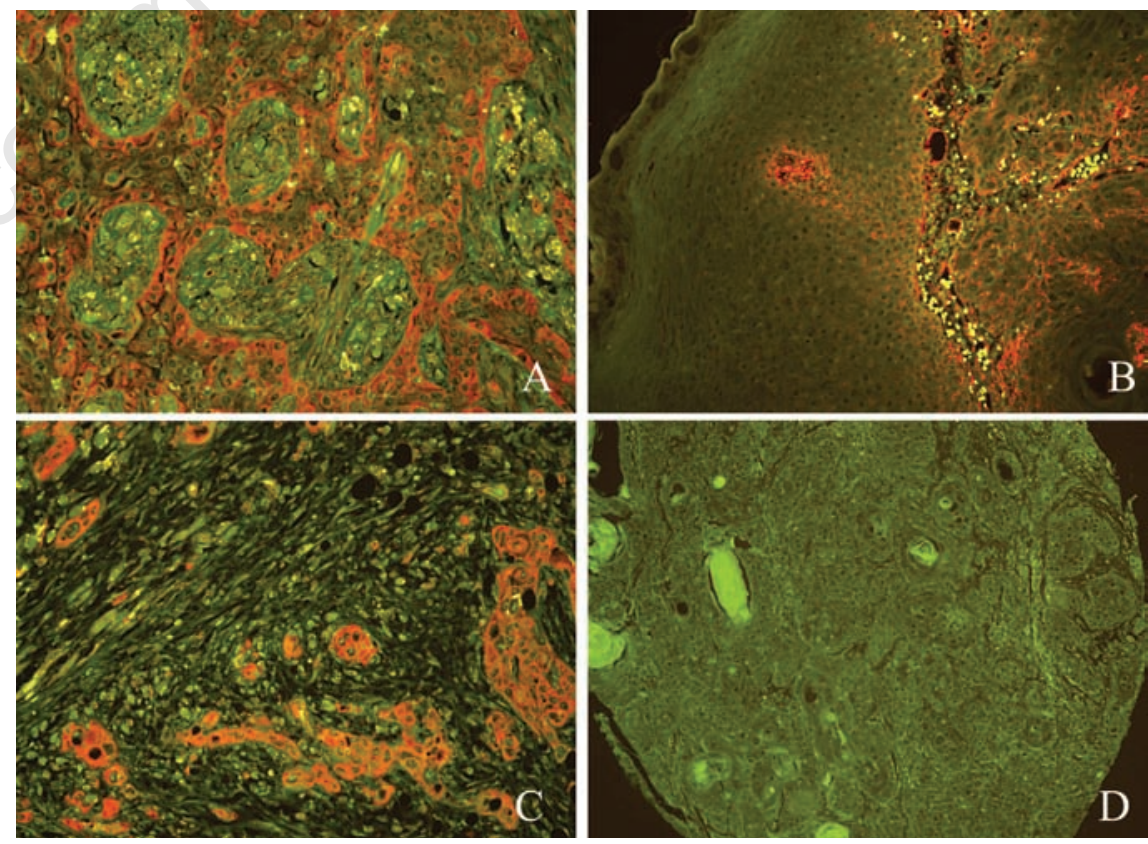

Figure 2. Cav-1 expression by QDs-IHC in PTSCC. (A) Tumor cells in PTSCC positive for Cav-1 were obtained in a fine, granular pattern at the surface membrane and the cytoplasm; in the peripheral cancer nests were observed stronger positive signals than in the middle cancer nests $(\mathrm{QDs}-\mathrm{IHC} \times 400)$. (B) In the same section, the Cav-1 expression in tumor nests was more intensive than in dysplastic mucosa (QDs-IHC $\times 200)$. (C) Single tumor cell in PTSCC positive for Cav-1 could be clearly detected (QDs-IHC $\times 400)$. (D) Negative control showed negative reactivity for Cav-1 (QDs-IHC $\times 100)$. 
fin-embedded lung tissue. Our present data indicate that $\mathrm{Cav}-1$ expression with moderate to high levels using QDs-IHC in 74\% of TSCC; positive signals were strong and clear with clean background, indicating QDs-IHC might be an efficient method for protein detection in tongue samples.

Cav-1 is the principal component of caveolae, which are special invaginated microdomains of the plasma membrane present in most mammalian cells. ${ }^{18}$ It plays a key role in the regulation of several signal transduction molecules and attracts more and more attention due to its paradoxical biological functions in malignant tumors. In this study, we noted for the first time a progressive increase of Cav-1 expression from NTM, HTM, TPL to PTSCC (a series of tissues ranging from normal to neoplastic), which is a trend of increased expression of Cav-1 in the stepwise tongue carcinogenesis. Moreover, in the same sample the Cav-1 expression in tumor nests was more intensive than that in dysplasia mucosa. The up-regulated expression of Cav-1 observed in the present study using QDs-IHC was similar to that reported in studies of esophageal squamous cell carcinoma, ${ }^{4}$ prostate cancer ${ }^{5}$ and pancreatic cancer. ${ }^{7}$ It has been suggested that the secreted fraction of Cav-1 is associated with cell released microvesicles, which are able to confer tumorigenicity in vitro as well as in vivo. Our study showed that up-regulated Cav-1 expression was associated with advanced clinical stage and high-grade malignancy of TSCC, while Nakatani et al. ${ }^{9}$ found that the level of Cav-1 expression did not differ among stages or other clinical parameters, except for the level of Cav-1 expression between the well-differentiated and poorly differentiated groups. The reasons might be as follows: i) different methods; our group just used a different method in the present study. ii) different samples; Nakatani et al. ${ }^{9}$ used OSCC samples in different sites including tongue, maxilla, mandibular and floor of mouth, while all of our samples arised from the tongue. iii) a different score system for the immunoevaluation used in the present study. In addition, in the present research, in PTSCC were observed stronger positive signals in the peripheral cancer nests than in the middle cancer nests; this is in agreemetn with the results of previous studies, ${ }^{19,20}$ showing that Cav-1 is preferentially expressed in tumor cells with basal-like immunophenotype, as defined by cDNA microarrays or immunohistochemistry.

On the other hand, a previous study ${ }^{10}$ disclosed that the inactivation of Cav-1 by a mutation or by reduced expression might play a role in the pathogenesis of oral cancer. Some authors also found that Cav-1 might be a tumor suppressor, because of its decreased expres-
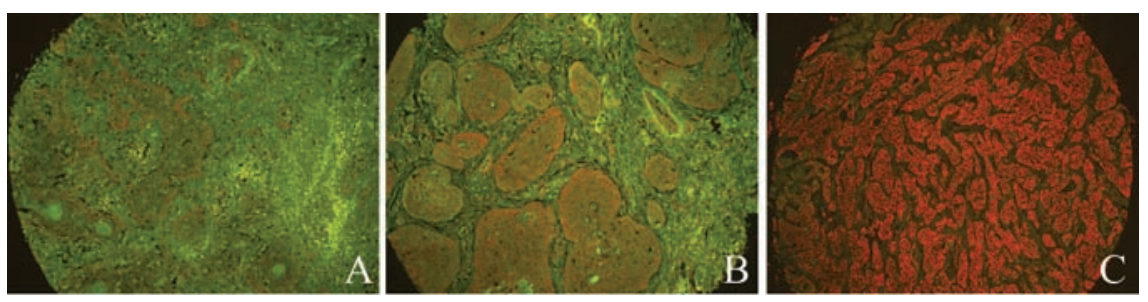

Figure 3. Cav-1 expression in PTSCC with different histological degree by QDs-IHC. (A) Tumor cells in PTSCC with high degree of histological differentiation showed weak positivity for Cav-1 (QDs-IHC $\times 100)$. (B) Tumor cells in PTSCC with moderate degree of histological differentiation showed moderate positivity for Cav-1 (QDs-IHC $\times 100)$. (C) Tumor cells in PTSCC with low degree of histological differentiation showed intensive positivity for Cav-1 (QDs-IHC $\times 100)$.

sion in a variety of cancer such as breast carcinoma, colon carcinoma, uterine cervical carcinoma, sarcoma of head and neck. ${ }^{21}$ Lee and colleagues $^{22}$ suggested that the diverse effects of Cav-1 might be simply mediated by the different regions of Cav-1 molecule and might be dependent on the levels of other molecules that are co-expressed with Cav-1.

Although the sample size for tongue epithelial dysplasia in the current study is insufficient for statistical analysis on Cav-1 expression, 10 "mild" to "moderate" tongue epithelial dysplasia revealed basal or parabasal cells staining, whereas 5 "severe" tongue epithelial dysplasia samples revealed the whole epithelial layer staining. This staining pattern of upward extension of Cav-1 protein may suggest that the Cav-1 expression was consistent with the severity of oral epithelial dysplasia.

In conclusion, our results may suggest that Cav-1 protein is one of the oncogenes that contribute to the carcinogenesis and development of TSCC. Whether Cav-1 is an important predictor or prognosis for survival still awaits the extension of clinical follow-up.

\section{References}

1. Shah JP, Lydiatt W. Treatment of cancer of the head and neck. Cancer J Clin 1995;45: 352-368.

2. Perrone G, Altomare V, Zagami M, Morini S, Petitti T, Battista C et al. Caveolin-1 expression in human breast lobular cancer progression. Mod Pathol 2009;22:71-8.

3. Sunaga N, Miyajima K, Suzuki M, Sato M, White MA, Ramirez RD et al. Different roles for caveolin-1 in the development of non-small cell lung cancer versus small cell lung cancer. Cancer Res 2004;64:427742.

4. Ando T, Ishiguro H, Kimura M, Mitsui A, Mori Y, Sugito $\mathrm{N}$ et al. The overexpression of caveolin-1 and caveolin-2 correlates with a poor prognosis and tumor progression in esophageal squamous cell carcinoma. Oncol Rep 2007; 18:601-9.

5. Karam JA, Lotan Y, Roehrborn CG, Ashfaq R, Karakiewicz PI, Shariat SF. Caveolin-1 overexpression is associated with aggressive prostate cancer recurrence. Prostate 2007;67:614-22.

6. Ito Y, Yoshida H, Tomoda C, Uruno T, Takamura Y, Miya A et al. Caveolin-1 and 14-3-3 sigma expression in follicular variant of thyroid papillary carcinoma. Pathol Res Pract 2005;201:545-9.

7. Tanase CP, Dima S, Mihai M, Raducan E, Nicolescu MI, Albulescu L et al. Caveolin-1 overexpression correlates with tumour progression markers in pancreatic ductal adenocarcinoma. J Mol Histol 2009; 40:239.

8. Hung KF, Lin SC, Liu CJ, Chang CS, Chang KW, Kao SY. The biphasic differential expression of the cellular membrane protein, caveolin-1, in oral carcinogenesis. $\mathrm{J}$ Oral Pathol Med 2003;32:461-7.

9. Nakatani K, Wada T, Nakamura M, Uzawa K, Tanzawa H, Fujita S. Expression of caveolin-1 and its correlation with cisplatin sensitivity in oral squamous cell carcinoma. J Cancer Res Clin Oncol 2005;131:44552.

10. Han SE, Park KH, Lee G, Huh YJ, Min BM. Mutation and aberrant expression of Caveolin-1 in human oral squamous cell carcinomas and oral cancer cell lines. Int $\mathrm{J}$ Oncol 2004; 24:435-40.

11. Liu Z, Cai WB, He LN, Nakayama N, Chen $\mathrm{K}$, Sun XM et al. In vivo biodistribution and highly efficient tumour targeting of carbon nanotubes in mice. Nat Nanotechol 2007;2:47-52.

12. Smith AM, Dave $S$, Nie $S$, True L, Gao X. Multicolor quantum dots for molecular diagnostics of cancer. Expert Rev Mol Diagn 2006;6:231-44.

13. Sobin LH, Wittekind $\mathrm{CH}$. International Union Against Cancer TNM classification 
of malignant tumours, 5th edn. Wiley, New York, 1997.

14. Vigneswaran N, Zhao W, Dassanayake A Muller S, Miller DM, Zacharias W. Variable expression of cathepsin B and D correlates with highly invasive and metastatic phenotype of oral cancer. Hum Pathol 2000; 31:931-7.

15. Byers RJ, Di Vizio D, O'connell F, Tholouli E, Levenson RM, Gossage K et al. Semiautomated multiplexed quantum dotbased in situ hybridization and spectral deconvolution. J Mol Diagn 2007;9:20-9

16. Xing Y, Chaudry Q, Shen C, Kong KY, Zhau HE, Chung LW et al. Bioconjugated quantum dots for multiplexed and quantitative immunohistochemistry. Nat Protoc 2007;2:1152-65.

17. Chen H, Xue J, Zhang Y, Zhu X, Gao J, Yu
B. Comparison of quantum dots immunofluorescence histochemistry and conventional immunohistochemistry for the detection of caveolin-1 and PCNA in the lung cancer tissue microarray. J Mol Histol 2009;40:261-8

18. Anderson RG, Jacobson K. A role for lipid shells in targeting proteins to caveolae, rafts, and other lipid domains. Science 2002;296:1821-5.

19. Pinilla SM, Honrado E, Hardisson D, Benitez J, Palacios J. Caveolin-1 expression is associated with a basal-like phenotype in sporadic and hereditary breast cancer. Breast Cancer Res Treat 2006;99:8590.

20. Savage K, Lambros MB, Robertson D, Jones RL, Jones C, Mackay A et al. Caveolin 1 is overexpressed and amplified in a subset of basal-like and metaplastic breast carcinomas: a morphologic, ultrastructural, immunohistochemical, and in situ hybridization analysis. Clin Cancer Res 2007;13:90-101.

21. Zhang H, Su L, Müller S, Tighiouart M, Xu Z, Zhang X et al. Restoration of caveolin-1 expression suppresses growth and metastasis of head and neck squamous cell carcinoma. Br J Cancer 2008;99:1684-94.

22. Lee H, Volonte D, Galbiati F, Iyengar $P$, Lublin DM, Bregman DB et al. Constitutive and growth factor-regulated phosphorylation of caveolin-1 occurs at the same site (Tyr-14) in vivo: identification of a cSrc/Cav-1/Grb7 signaling cassette. Mol Endocrinol 2000;14:1750-75. 